\title{
Effects of incorrect detrending on the coherency between non-stationary time series processes
}

\author{
Jin Lee ${ }^{1, a}$ \\ ${ }^{a}$ Department of Economics, Ewha Womans University, Korea
}

\begin{abstract}
We study the effect of detrending on the coherency between two time series processes. Many economic and financial time series variables include nonstationary components; however, we analyze the two most popular cases of stochastic and deterministic trends. We analyze the asymptotic behavior of coherency under incorrect detrending, which includes the cases of first-differencing the deterministic trend process and, conversely, the time trend removal of the unit root process. A simulation study is performed to investigate the finite sample performance of the sample coherency due to incorrect detrending. Our work is expected to draw attention to the possible distortion of coherency when the series are incorrectly detrended. Further, our results can extend to various specification of trends in aggregate time series variables.
\end{abstract}

Keywords: coherency, stochastic trends, deterministic trends, detrending

\section{Introduction}

Specifications of trends in nonstationary time series processes have been an issue in wide areas of economics literature since the early 1980s (Nelson and Kang, 1981; Nelson and Plosser, 1982; Murray and Nelson, 2000; Perron and Wada, 2009). In an economics context, stochastic trends and deterministic trends are the two most popular types. Therefore, detrending methods typically include time trend removal and first-differencing. Early literature focused on the effects of the misspecification of trends on the behavior of spectral densities (e.g., Harvey and Jaeger, 1993; Canova, 1998; Fleissig and Strauss, 1999; Aadland, 2005). We also note that Dagum and Giannerini (2006) investigate the effects of the misspecification of trends on various hypothesis tests that include tests for stationarity and tests for linearity. Ashley and Verbrugge (2006) study how parameter estimates are vulnerable to detrending methods. These works have drawn attention to studies on the distortions of various statistics due to incorrect detrending.

In this work, we consider coherency, which is a correlation measure in the frequency domain. Pearson type correlations defined in time domain compute the comovement between the series at different lags; however, coherency measures comovement between the time series at different frequency, as it is based on the spectral representation of time series. Thus, one can obtain long-run, mediumrun or short-run correlations between the series, which generate complementary implications from standard auto-correlations in time domain.

We study how coherence measures are affected by detrending methods. In addition, we pay attention to stochastic and deterministic trends as well as analyze the effect of incorrect detrending on

\footnotetext{
${ }^{1}$ Department of Economics, Ewha Womans University, 52 Ewhayeodae-Gil, Seodaemun-Gu, Seoul 03760, Korea.

E-mail: leejin@ewha.ac.kr
}

Published 31 January 2019 / journal homepage: http://csam.or.kr

(c) 2019 The Korean Statistical Society, and Korean International Statistical Society. All rights reserved. 
the behavior of coherency, both theoretically and numerically. First, the limiting form of coherency under correct and incorrect detrending is studied. Next, some simulation works are presented to see the effect of detrending on the finite sample performance of sample coherency. We also present a brief empirical work using foreign exchange rate data. Section 5 concludes the study.

\section{Effects of false detrending on asymptotics of coherency}

We consider a modified coherency (MC), proposed by Croux et al. (2001), which is the real part of the coherency measures. Write

$$
\rho_{x y}(\lambda)=\frac{f_{x y}(\lambda)}{\left(f_{x}(\lambda) f_{y}(\lambda)\right)^{\frac{1}{2}}}, \quad \text { for } \lambda \in[-\pi, \pi],
$$

where $f_{x y}(\lambda)$ is the cospectrum, which equals the real part of the cross spectral density; additionally, and $f_{x}(\lambda)$ and $f_{y}(\lambda)$ are the auto-spectral densities of covariance-stationary $x$ and $y$, respectively (e.g., Priestley, 1981).

We call this a MC when the usual coherency is complex-valued. In order for the MC to be welldefined, the underlying processes need to be second-order stationary. If the data contains nonstationary components, appropriate detrending is required. We therefore consider two types of nonstationary cases of stochastic trends (or unit roots) and deterministic trends. Below, we consider two cases of false detrending.

First we consider the case that true processes consist of stationary components around the deterministic time trend. In addition, stationary innovations are assumed to have a linear structures as in Phillips and Solo (1992). It helps construct the limiting forms of MC under correct and incorrect detrending. We formally introduce the following assumption,

Assumption 1. Bivariate series $q_{t}=\left(x_{t}, y_{t}\right)^{\prime}$ follows trend stationary processes,

$$
q_{t}=\alpha+\tau t+e_{t}
$$

where $\alpha=\left(\alpha_{1}, \alpha_{2}\right)^{\prime}, \tau=\left(\tau_{1}, \tau_{2}\right)^{\prime}$, and $e_{t}=\left(e_{1 t}, e_{2 t}\right)^{\prime}$ is a linear process given by

$$
e_{t}=\phi(L) \varepsilon_{t}=\sum_{k=0}^{\infty} \phi_{k} \varepsilon_{t-k}=\sum_{k=0}^{\infty}\left(\begin{array}{ll}
a_{k} & b_{k} \\
c_{k} & d k
\end{array}\right)\left(\begin{array}{l}
\varepsilon_{1 t-k} \\
\varepsilon_{2 t-k}
\end{array}\right),
$$

where $\varepsilon_{t}=\left(\varepsilon_{1 t}, \varepsilon_{2 t}\right)^{\prime}$ is iid $\left(0, \sigma^{2} I_{2}\right), \sum_{k=0}^{\infty} k^{\delta}\left\|\phi_{k}\right\|<\infty$, for $\delta \geq 1$ with $\left\|\phi_{k}\right\|=\left[\sum_{k}\left|\phi_{k}^{i, j}\right|^{2}\right]^{1 / 2}$, for $(i, j)=(1,1), \ldots,(2,2)$.

The linear structure of innovations given in the assumption is standard where long-run and shortrun components are compactly expressed through Beveridge-Nelson decomposition techniques (Phillips and Solo, 1992).

In relation to the variance and covariance structures of linear processes given above, we define the following quantities.

$$
f_{j}^{11}(L)=\sum_{k=0}^{\infty} a_{k} a_{k-j} L^{k}, \quad f_{j}^{12}(L)=\sum_{k=0}^{\infty} b_{k} b_{k-j} L^{k},
$$




$$
\begin{aligned}
f_{j}^{21}(L) & =\sum_{k=0}^{\infty} c_{k} c_{k-j} L^{k}, & f_{j}^{22}(L) & =\sum_{k=0}^{\infty} d_{k} d_{k-j} L^{k}, \\
g_{j}(L) & =\sum_{k=0}^{\infty} a_{k} c_{k-j} L^{k}, & h_{j}(L) & =\sum_{k=0}^{\infty} b_{k} d_{k-j} L^{k} .
\end{aligned}
$$

Under the linear structure of the innovations, we can derive the explicit form of the MC, as follows. The following theorem states the effects of correct and incorrect detrending on the asymptotics of MC.

Theorem 1. Under the Assumption 1,

(i) The MC for time-detrended $q_{t}$ equals

$$
\rho_{x y}^{*}(\lambda)=\frac{f_{12}^{*}(\lambda)}{\left[f_{1}^{*}(\lambda) f_{2}^{*}(\lambda)\right]^{\frac{1}{2}}},
$$

where $f_{1}^{*}(\lambda)=\sum_{j=-\infty}^{\infty}\left(f_{j}^{11}(1)+f_{j}^{12}(1)\right) \cos (j \lambda), f_{2}^{*}(\lambda)=\sum_{j=-\infty}^{\infty}\left(f_{j}^{21}(1)+f_{j}^{22}(1)\right) \cos (j \lambda)$, and $f_{12}^{*}(\lambda)=\sum_{j=-\infty}^{\infty}\left(g_{j}(1)+h_{j}(1)\right) \cos (j \lambda)$.

(ii) The MC of first-differenced $\Delta q_{t}$ equals

$$
\rho_{x y}(\lambda)=\frac{m_{12}(\lambda)}{\left[m_{1}(\lambda) m_{2}(\lambda)\right]^{\frac{1}{2}}},
$$

where $m_{1}(\lambda)=\sum_{j=-\infty}^{\infty} f_{1 j} \cos (j \lambda), m_{2}(\lambda)=\sum_{j=-\infty}^{\infty} m_{2 j} \cos (j \lambda)$, and $m_{12}(\lambda)=\sum_{j=-\infty}^{\infty} f_{12 j} \cos (j \lambda)$, with

$$
\begin{aligned}
f_{1 j} & =2\left(f_{j}^{11}(1)+f_{j}^{12}(1)\right)-f_{j-1}^{11}(1)-f_{j+1}^{11}(1)-f_{j-1}^{12}(1)-f_{j+1}^{12}(1), \\
f_{2 j} & =2\left(f_{j}^{21}(1)+f_{j}^{22}(1)\right)-f_{j-1}^{21}(1)-f_{j+1}^{21}(1)-f_{j-1}^{22}(1)-f_{j+1}^{22}(1), \\
f_{12 j} & =g_{j}(1)+h_{j}(1)+2\left(g_{j-1}(1)+h_{j-1}(1)\right) .
\end{aligned}
$$

The part (i) refers to the correct specification of trend, where the superscript * refers to the correct quantity. However, the part (ii) arises from misspecification of trend. We only prove part(ii).

Proof of Theorem 1: The proof is based on Phillips and Solo (1992, eq.28) and Maynard and Shimotsu (2009). For the differenced processes, the auto-covariances are written as $E\left(\Delta \varepsilon_{t} \Delta \varepsilon_{t-r}\right)=-\sigma^{2}$, for $r \neq 0$, and for $|r|>1, E\left(\Delta \varepsilon_{t} \Delta \varepsilon_{t-r}\right)=0$. We then obtain

$$
\begin{aligned}
E\left(\Delta e_{1 t} \Delta e_{1 t-j}\right)= & f_{j}^{11}(1) E\left(\Delta \varepsilon_{1 t}^{2}\right)+\sum_{r=1}^{\infty}\left[f_{j-r}^{11}(1) E\left(\Delta \varepsilon_{1 t} \varepsilon_{1 t-r}\right)+f_{j+r}^{11}(1) E\left(\Delta \varepsilon_{1 t} \Delta \varepsilon_{1 t+r}\right)\right] \\
& +f_{j}^{12}(1) E\left(\Delta \varepsilon_{2 t}^{2}\right)+\sum_{r=1}^{\infty}\left[f_{j-r}^{12}(1) E\left(\Delta \varepsilon_{2 t} \Delta \varepsilon_{2 t-r}\right)+f_{j+r}^{12}(1) E\left(\Delta \varepsilon_{2 t} \Delta \varepsilon_{2 t+r}\right)\right] \\
= & \sigma^{2}\left[2\left(f_{j}^{11}(1)+f_{j}^{12}(1)\right)-f_{j-1}^{11}(1)-f_{j+1}^{11}(1)-f_{j-1}^{12}(1)-f_{j+1}^{12}(1)\right] .
\end{aligned}
$$

Similarly, we get

$$
E\left(\Delta e_{2 t} \Delta e_{2 t-j}\right)=\sigma^{2}\left[2\left(f_{j}^{21}(1)+f_{j}^{22}(1)\right)-f_{j-1}^{21}(1)-f_{j+1}^{21}(1)-f_{j-1}^{22}(1)-f_{j+1}^{22}(1)\right] .
$$


For the cross covariance and the cospectrum, we use the results in Maynard and Shimotsu (2009, Lemma 13) to get $E\left(\Delta e_{1 t} \Delta e_{2 t-j}\right)=\sigma^{2}\left[g_{j}(1)+h_{j}(1)+2\left(g_{j-1}(1)+h_{j-1}(1)\right)\right]$.

The results of the part (ii) come from the case that trend stationary processes are first-differenced, which entail over-differenced series. This type of misspecification is also known as the moving average (MA) unit root. Since over-differenced series generate additional correlations, the forms of autospectrum and cospectrum become more complicated than a correctly detrended case. Consequently, the modified MC due to incorrect detrending of generated inaccurate values.

Remark 1. To further get an insight for the MA unit root problem, we consider a simple example that $a_{0}=b_{0}=1$ and $a_{k}=b_{k}=0$ for $k>0$ in the process of $e_{t}$. It then follows that $E\left(\Delta e_{1 t}^{2}\right)=2 \sigma^{2}$, and $E\left(\Delta e_{1 t} \Delta e_{1 t-1}\right)=E\left(\Delta e_{1 t} \Delta e_{1 t+1}\right)=-\sigma^{2}$. Then, the auto-spectral density of $e_{1 t}$ becomes

$$
f_{1}(\lambda)=2(1-\cos (\lambda))
$$

It is then noted that degeneracy arises at the zero frequency, i.e., $f_{1}(0)=0$. Thus, false detrending invalidates the $\mathrm{MC}$ at the zero frequency.

We next analyze the converse case of misspecification of trend, where stochastic trends are mistakenly treated as a time trend process. In this case, the coherency is not well defined, since nonstationary components remain in the underlying process. Thus, we treat this case with less weight, compared to the previous case. We formally consider the assumptions for unit root processes.

Assumption 2. Bivariate series $q_{t}=\left(x_{t}, y_{t}\right)^{\prime}$ follow unit root processes with a drift, $q_{t}=\alpha+q_{t-1}+e_{t}$, where innovations $e_{t}$ are specified as in part(ii) in Assumption 1.

The Assumptions 2 is one of the most popular unit root specification in economics context, though rather simplistic. Under unit root assumption, $q_{t}=\alpha t+\sum_{j=1}^{t} e_{j}$. Then, the $q_{t}$ is still nonstationary (i.e., the second moments do not exist) after time trend removal and as a result, spectral densities as well as the coherency are not defined. Below, we consider the case that deterministic terms are completely removed for heuristic purpose. Given this, we use $I(1)$ asymptotic theories to verify the asymptotic behavior of the quantity given by

$$
f_{x T}=(2 \pi)^{-1} \sum_{j=-\infty}^{\infty} R_{x}(j)
$$

where $R_{x}(j)=T^{-1} \sum_{t=|j|+1}^{T} h_{1 t} h_{1 t-|j|}$ for $h_{t}=q_{t}-\alpha t=\left(h_{1 t}, h_{2 t}\right)^{\prime}$. By invariance principle (Phillips and Solo, 1992), we obtain

$$
T^{-\frac{1}{2}} h_{t}=T^{-\frac{1}{2}} \sum_{j=1}^{t=[r T]} e_{j} \rightarrow U(r)
$$

for $r \in[0,1]$, where $U(r)=\left(U_{1}(r), U_{2}(r)\right)^{\prime}$ denotes bivariate Brownian motions with $E\left(e_{1 t}^{2}\right)=\sigma_{1}^{2}$, $E\left(e_{2 t}^{2}\right)=\sigma_{2}^{2}$, and $E\left(e_{1 t} e_{2 t}\right)=\sigma_{12}$. We then obtain the limit,

$$
T^{-2} \sum_{j=-\infty}^{\infty} R_{x}(j) \rightarrow\left[\int_{0}^{1} U_{1}(r) d r\right]^{2},
$$


thereby, we get $T^{-2} f_{T} \rightarrow(2 \pi)^{-1}\left[\int_{0}^{1} U_{1}(r) d r\right]^{2}$.

The same reasoning applies to

$$
f_{y T}=(2 \pi)^{-1} \sum_{j=-\infty}^{\infty} R_{y}(j), \quad f_{x y T}=(2 \pi)^{-1} \sum_{j=-\infty}^{\infty} R_{x y}(j),
$$

where

$$
R_{y}(j)=T^{-1} \sum_{t=|j|+1}^{T} h_{2 t} h_{2 t-|j|}, \quad R_{x y}(j)=T^{-1} \sum_{t=|j|+1}^{T} h_{1 t} h_{2 t-|j|} .
$$

Thus, both numerator and denominator are divergent with the order of $O_{p}\left(T^{2}\right)$. These results are summarized as follows.

Lemma 1. Suppose the Assumption 2 holds and the deterministic trends are completely detrended. Then, the following ratio converges to limit, as $T \rightarrow \infty$,

$$
\frac{f_{x y T}}{\left(f_{x T} f_{y T}\right)^{\frac{1}{2}}} \rightarrow \frac{\int_{0}^{1} U_{1}(r) U_{2}(r) d r}{\left[\left\{\int_{0}^{1} U_{1}(r) d r\right\}^{2}\left\{\int_{0}^{1} U_{2}(r) d r\right\}^{2}\right]^{\frac{1}{2}}},
$$

Remark 2. The Lemma 1 only provides a heuristic inference. If the deterministic trend $\alpha$ in the Assumption 2 is not completely removed, then the trends dominate the partial sum of errors. Then, the resulting inferences become complicated and unrelated with coherency measures. Thus, we do not extend further analysis. However, more complicated forms of unit root processes (e.g., local-to-unity) than the simple form in Assumption 1 could be considered in subsequent research.

\section{Simulation studies}

In this section, we conduct a small set of simulation studies to see the effect of correct and incorrect detrending on coherency. For coherency estimates, we use kernel-based nonparametric estimators for auto-spectral density and cospectrum. The auto-spectral densities of the covariance-stationary variable $x$ is given by

$$
f_{x}(\lambda)=R_{x}(0)+2 \sum_{j=1}^{T-1} k\left(\frac{j}{M}\right) R_{x}(j) \cos (2 \pi \lambda),
$$

where $\lambda \in(0,2 \pi)$ is a frequency, $k$ is a kernel function, $M$ is the lag truncation number (bandwidth). The sample variances are given by $R_{x}(j)=T^{-1} \sum_{t=|j|+1}^{T}\left(x_{t}-\bar{x}\right)\left(x_{t-|j|}-\bar{x}\right)$, with $\bar{x}$ is the sample mean of $x$.

Also, the cospectrum estimator is given by

$$
f_{x y}(\lambda)=\sum_{j=1-T}^{T-1} k\left(\frac{j}{M}\right) R_{x y}(j) \cos (2 \pi \lambda),
$$

where the sample cross covariance equals to $R_{x y}(j)=T^{-1} \sum_{t=|j|+1}^{T}\left(x_{t}-\bar{x}\right)\left(y_{t-|j|}-y\right)$, and it is noted that the cross covariance is not symmetric in $j$. 
Table 1: Bias and MSE of coherency estimates: true DGP = linear time trend

\begin{tabular}{ccrr}
\hline \hline AR & Frequency & Bias(MSE)-first differencing & Bias(MSE)-time removal \\
\hline & 0.01 & $0.1407(0.0325)$ & $0.0003(0.0203)$ \\
$\beta=0.0$ & 0.05 & $0.1018(0.0157)$ & $0.0006(0.0159)$ \\
& 0.1 & $0.0362(0.0127)$ & $0.0017(0.0110)$ \\
& 0.5 & $-0.0048(0.0208)$ & $-0.0050(0.0184)$ \\
$\beta=0.5$ & 1.0 & $0.1414(0.0329)$ & $0.0003(0.0203)$ \\
& 0.01 & $0.1120(0.0244)$ & $0.0004(0.0264)$ \\
& 0.05 & $0.0788(0.0153)$ & $0.0005(0.0224)$ \\
& 0.1 & $0.0279(0.0125)$ & $0.0010(0.0142)$ \\
& 0.5 & $-0.0033(0.0195)$ & $-0.0036(0.0149)$ \\
\hline \hline
\end{tabular}

MSE $=$ mean squared errors; $\mathrm{DGP}=$ data generating process; AR $=$ auto-regressive.

The bandwidth $M$ is required to satisfy the condition that $M \rightarrow \infty$ and $M / T \rightarrow 0$, which guarantees consistency of the estimators (e.g., Priestley, 1981; Andrews 1991). In our simulation, we set $M=\left[4(T / 100)^{2 / 9}\right]$, as used in Kwiatkowski et al. (1992). One can use parametric or nonparametric bandwidth choice rules in line with the context of heteroskedasticity and autocorrelation consistent (HAC) covariance matrix estimation. Alternatively, we use a simple rule for the bandwidth to reduce the computational burden.

First, we consider a linear time trend process as the data generating process (DGP). Let $q_{t}=$ $\left(x_{t}, y_{t}\right)^{\prime}$ be generated by

$$
\text { DGP } 1: q_{t}=\mu+\delta t+e_{t},
$$

where $\mu=\left(\mu_{1}, \mu_{2}\right)^{\prime}, \delta=\left(\delta_{1}, \delta_{2}\right)^{\prime}$. Error terms $e_{t}=\left(e_{1 t}, e_{2 t}\right)^{\prime}$, where $e_{i t}=\beta e_{i t}+\varepsilon_{i t}$, and $\varepsilon_{i t}$ are iid $N(0,1)$, with $E\left(\varepsilon_{i t}\right)=0, E\left(\varepsilon_{i t}^{2}\right)=1$, for $i=1,2$, and $E\left(\varepsilon_{1 t} \varepsilon_{2 s}\right)=0$ for all $t, s$.

The parameters are set as $\mu_{1}=\mu_{2}=0, \delta_{1}=\delta_{2}=0.1$, and $\beta=0$ or 0.5 .

Given the above setup, one can obtain the theoretical expressions for the auto, cross spectrum of correctly detrended series, i.e.,

$$
f_{x}(\lambda)=f_{y}(\lambda)=(2 \pi)^{-1}\left(1-2 \rho \cos (\lambda)+\rho^{2}\right)^{-1},
$$

and $f_{x y}(\lambda)=0$. It follows that the true value of MC at the origin, $\rho_{x y}(\lambda)=0$.

We conduct 1,000 iterations and compute the bias and the mean squared errors (MSE), where MSE is computed as $(1 / B) \sum_{m=1}^{B}\left[\hat{\rho}_{m, x y}(\lambda)-\rho_{x y}(\lambda)\right]^{2}$, for the true coherency $\rho_{x y}(\lambda)$ and the number of iterations $B$. The pre-specified frequencies are set from $\lambda=0.01$ to 1 . In the economic context, small values of frequencies draw attention as they are related to the long-run information.

Table 1 shows the results when the true DGP is a linear time trend process. Two different autoregressive (AR) processes for innovations with $\beta=0$ and 0.5 are included. The first differencing, with a very few exceptions, yields a larger bias than the case of time trend removal. Incorrect detrending cause notable bias particularly at frequencies of $0.01,0.05$, and 1 . Such pattern of the bias remains unchanged when the AR parameter increases to 0.5. However, unlike the bias properties, two different MSEs do not dominate each other as they vary with frequency. Theorem 1 indicates that over-differenced series cause smaller variances than the correctly detrended series. Therefore, we only note that the bias problems are a distortion due to false detrending.

Next, we consider unit root with a drift processes for $q_{t}$,

$$
\text { DGP 2: } q_{t}=\alpha+q_{t-1}+e_{t}
$$


Table 2: Bias and MSE of coherency estimates: True DGP = unit root with a drift

\begin{tabular}{cccc}
\hline \hline AR & Frequency & Bias(MSE)-first differencing & Bias(MSE)-time removal \\
\hline & 0.01 & $0.0517(0.0238)$ & $-0.0068(0.2048)$ \\
$\beta=0$ & 0.05 & $0.0437(0.0186)$ & $-0.0068(0.2017)$ \\
& 0.1 & $0.0227(0.0117)$ & $-0.0063(0.1853)$ \\
& 0.5 & $-0.0017(0.0185)$ & $-0.0066(0.2016)$ \\
$\beta=0.5$ & 1.0 & $0.0518(0.0238)$ & $-0.0068(0.2048)$ \\
& 0.01 & $0.0165(0.0269)$ & $-0.0070(0.2099)$ \\
& 0.05 & $0.0149(0.0229)$ & $-0.0070(0.2076)$ \\
& 0.1 & $0.0097(0.0144)$ & $-0.0067(0.1955)$ \\
& 0.5 & $0.0019(0.0159)$ & $-0.0070(0.2247)$ \\
& 1.0 & $0.0166(0.0269)$ & $-0.0070(0.2099)$ \\
\hline \hline
\end{tabular}

$\mathrm{MSE}=$ mean squared errors; DGP $=$ data generating process; $\mathrm{AR}=$ auto-regressive .

where $\alpha=\left(\alpha_{1}, \alpha_{2}\right)^{\prime}$ and $e_{t}=\left(e_{1 t}, e_{2 t}\right)^{\prime}$, as in the DGP 1. The drift terms are set as $\alpha_{1}=\alpha_{2}=0.1$.

Therefore, it is known that unit roots with a drift behave as a linear time trend process. Table 2 shows that correct first-differencing clearly dominates the incorrect time removal in terms of MSEs. Time removal yields nearly eight to ten times larger MSEs than first-differencing. In terms of bias property, time removal generates a smaller bias, even though it is an incorrect detrending. The bias properties may require more extensive simulations. Therefore, it is inferred that a significant increase of MSE in incorrect detrending comes from the inflated variance of the coherency estimates. The MSEs by correct detrending remain stable when the AR coefficient increases.

Other choices of AR parameters and correlation parameters between innovations yield qualitatively analogous results, which are omitted to save space. In addition, alternative DGP specifications are left for future research.

\section{Empirical studies}

We perform an empirical study using real data and investigate the effect of detrending on coherency measures between two variables. In doing so, we consider two foreign exchange rates-euro/dollar exchange rate and dollar/pound exchange rate. The data has 284 monthly observations, which range from January, 1995 to August, 2018. The data is available from the Bank of Korea dataset.

First, we conduct unit root tests for exchange rates. Two testing procedures of Dickey-Fuller (DF) and variance ratio (VR) by Breitung (2002) are been applied. For the DF test, the 5\% critical value equals to -2.88 in the presence of intercept and a linear trend. The test values are -2.0029 and -2.01 for euro/dollar rate and dollar/pound rate, respectively. Thus, the null hypothesis of unit roots are not rejected at the 5\% significance level. As for the VR test, we compute the test values of 0.0223 and 0.017 for euro/dollar and dollar/pound rates, respectively. As the 5\% left-tailed critical value is given as 0.01 , the null hypothesis of unit roots are not rejected. Therefore, both test results indicate that the these exchange rates are highly likely to follow unit root processes.

The test results allow us to compute the coherency based on two different detrending methodsfirst differencing and trend removal. First differenced series yield the estimated coherency between the two exchange rates as $0.6718,0.6559,0.6053$, and 0.672 , at frequencies of $0.01,0.05,0.1$, and 1 , respectively. However, by trend removal, coherency is estimated as $0.9799,0.9798,0.9791$, and 0.9799 at frequencies of $0.01,0.05,0.1$, and 1 , respectively. Thus, it shows that two detrending methods generate a sharp contrast for the correlations in the frequency domain. Our empirical results imply a need to observe detrending the series in practical studies, even though the true trends are unknown. Data and computing coeds are available from the author by request. 


\section{Conclusion}

We analyze the effect of detrending on the coherency between two trending time series processes. Two popular types of trends of stochastic and deterministic trends are considered, and we study the behavior of coherency under incorrect detrending. A set of simulation studies are conducted to investigate the finite sample performance of the sample coherency due to correct and incorrect detrending. We also present a real data analysis using foreign exchange rates and show a significant difference by the two detrending methods. Correct detrending cannot be emphasized enough in practical research; therefore, our work is expected to draw attention to the possible distortion of coherency when the series are incorrectly detrended.

\section{References}

Aadland D (2005). Detrending time-aggregated data, Economics Letters, 9, 287-293.

Andrews DWK (1991). Heteroskedasticity and autocorrelation consistent covariance matrix estimation, Econometrica, 59, 817-858.

Ashley R and Verbrugge R (2006). Comments on "A critical investigation on detrending procedures for non-linear processes", Journal of Macroeconomics, 28, 192-194.

Breitung J (2002). Comments on "Nonparametric tests for unit roots and cointegration", Journal of Econometrics, 108, 343-363.

Canova F (1998). Detrending and business cycle facts, Journal of Macroeconomics, 41, 475-512.

Croux C, Forni M, and Reichlin L (2001). A measure of comovement for economic variables: theory and empirics, Review of Economics and Statistics, 83, 232-241.

Dagum E and Giannerini S (2006). A critical investigation on detrending procedures for non-linear processes, Journal of Macroeconomics, 28, 175-191.

Fleissig A and Strauss J (1999). Is OECD real per capita GDP trend or difference stationary? Evidence from panel unit root tests, Journal of Macroeconomics, 21, 673-690.

Harvey A and Jaeger A (1993). Detrending, stylized facts and the business cycle, Journal of Applied Econometrics, 8, 231-247.

Kwiatkowski D, Phillips PCB, Schmidt P, and Shin Y (1992). Testing the null of stationarity against the alternative of a unit root: how sure are we that economic time series have a unit root?, Journal of Econometrics, 54, 159-178.

Maynard A and Shimotsu K (2009). Covariance-based orthogonality tests for regressors with unknown persistence, Econometric Theory, 25, 63-116.

Murray C and Nelson C (2000). The uncertain trend in US GDP, Journal of Monetary Economics, 46, 79-95.

Nelson C and Kang H (1981). Spurious periodicity in inappropriate detrended time series, Econometrica, 49, 741-751.

Nelson C and Plosser C (1982). Trends and random walks in macroeconmic time series: some evidence and implications, Journal of Monetary Economics, 10, 139-162.

Perron P and Wada T (2009). Let's take a break: trends and cycles in US real GDP, Journal of Monetary Economics, 56, 749-765.

Phillips PCB and Solo V (1992). Asymptotics for linear processes, Annals of Statistics, 20, 971-1001. Priestley MB (1981). Spectral Analysis and Time Series, Academic Press, New York. 